\title{
P130 Cardiac Resynchronization Therapy Improves Early Ventricular Contraction and Reduces Time to Onset of Relaxation
}

\author{
Haotian $\mathrm{Gu}^{1, *}$, Simon Claridge ${ }^{2}$, Aldo Rinaldi ${ }^{2}$, Phil Chowienczyk ${ }^{1}$ \\ ${ }^{1}$ King's College London, London, UK \\ ${ }^{2}$ St Thomas' Hospital, London, UK
}

\section{ABSTRACT}

Background: Haemodynamic effects of Cardiac Resynchronisation Therapy (CRT) may be mediated by an improvement in early systolic function, as well as by improvements in overall systolic function. We examined whether CRT improves early ventricular contraction as measured by first-phase ejection fraction (EF1) and time to onset of ventricular relaxation (TOR).

Methods: EF1 was defined as the fraction of LV volume ejected from the start of systole to the time of the first systolic peak (T1) on the carotid pressure waveform. Time-varying ejection phase myocardial wall stress (MWS) was calculated from echocardiographic imaging of left ventricular volumes and left ventricular pressure was obtained by carotid tonometry during systole calibrated from peripheral mean and diastolic blood pressure. TOR was defined as time to peak MWS over ejection time.

Results: 25 patients (aged $72.8 \pm 8.5$ years) with heart failure and severely reduced EF $(30.2 \pm 7.0 \%)$ were studied before and immediately after CRT implantation. There was no difference in mean arterial pressure, central systolic pressure and augmentation pressure after CRT implantation. There was significant improvement in overall ejection fraction (pre: $30.2 \pm$ $6.7 \%$ vs post: $35.9 \pm 8.1 \%, p=0.002$ ) and $\mathrm{EF} 1$ (pre: $10.9 \pm 3.4 \%$ vs post: $13.7 \pm 4.7 \%, p=0.010$ ). TOR reduced significantly (pre: $48.0 \pm 14.3 \%$ vs post: $40.0 \pm 10.7 \%, p=0.0012)$. The change in EF1 was negatively associated with change in TOR $(\beta=-0.586$, $p=0.003)$. This relationship persisted after adjustment for age, gender, baseline MAP and baseline $\operatorname{EF} 1(\beta=-0.452, p=0.007)$.

Conclusion: In patients with heart failure, an improvement of early ejection is associated with reduction in TOR immediately after CRT implantation.

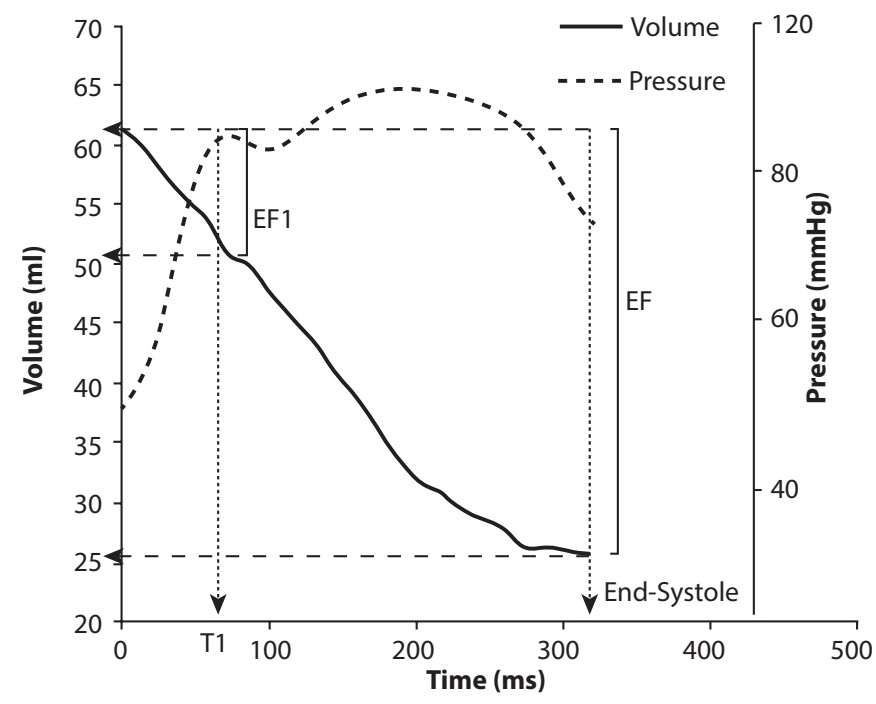

(c) 2019 Association for Research into Arterial Structure and Physiology. Publishing services by Atlantis Press International B.V. This is an open access article distributed under the CC BY-NC 4.0 license (http://creativecommons.org/licenses/by-nc/4.0/). 